\title{
Comparative efficacy and safety for different chemotherapy regimens used concurrently with thoracic radiation for locally advanced non-small cell lung cancer: a systematic review and network meta-analysis
}

\author{
Tingting Liư ${ }^{\dagger}$, Zheng $\mathrm{He}^{\dagger}$, Jun Dang ${ }^{*}$ (D) and Guang Li
}

\begin{abstract}
Background: It remains unknown which is the most preferable regimen used concurrently with thoracic radiation for locally advanced non-small cell lung cancer (NSCLC). We performed a network meta-analysis to address this important issue.

Methods: PubMed, Embase, Cochrane Library, Web of Science and major international scientific meetings were searched for relevant randomized controlled trials (RCTs). Overall survival (OS) data was the primary outcome of interest, and progression-free survival (PFS), and serious adverse events (SAEs) were the secondary outcomes of interests, reported as hazard ratio (HR) or odds ratio (OR) and 95\% confidence intervals (Cls).

Results: 14 RCTs with a total of 2975 patients randomized to receive twelve categories of treatments were included in the meta-analysis. Direct comparison meta-analysis showed that etoposide-cisplatin (EP) was more effective than paclitaxel-cisplatin/carboplatin (PC) in terms of OS ( $H R=0.85,95 \% \mathrm{Cl}: 0.77-0.94)$ and PFS (HR=0.66, 95\% Cl: $0.47-0$. 95). In network meta-analysis, all regimen comparisons did not produce statistically significant differences in survival. Based on treatment ranking of OS and the benefit-risk ratio, S-1-cisplatin (SP) was likely to be the most preferable regimen for its best efficacy and low risk of causing SAEs. Uracil/tegafur-cisplatin (UP) and pemetrexed-cisplatin/ carboplatin (PP) were ranked the second and third respectively. Gemcitabine-cisplatin (GP) and PC + Cetuximab (PCCet) appeared to be the worst and second-worst regimens for their poor efficacy and poor tolerability.

Conclusions: Based on efficacy and tolerability, SP is likely to be the most preferable regimen used concurrently with thoracic radiation for locally advanced NSCLC, followed by UP and PP. Further direct head-to-head studies are needed to confirm these findings.
\end{abstract}

Keywords: Locally advanced non-small cell lung cancer, Concurrent chemoradiation, Network meta-analysis

\footnotetext{
*Correspondence: dangjunsy@163.com

${ }^{\dagger}$ Tingting Liu and Zheng He contributed equally to this work.

Department of Radiation Oncology, The First Hospital of China Medical

University, 155 Nanjing Road, Heping District, Shenyang 110001, China
}

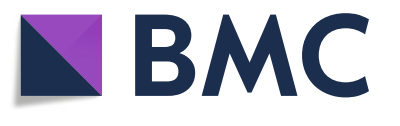

(C) The Author(s). 2019 Open Access This article is distributed under the terms of the Creative Commons Attribution 4.0 International License (http://creativecommons.org/licenses/by/4.0/), which permits unrestricted use, distribution, and reproduction in any medium, provided you give appropriate credit to the original author(s) and the source, provide a link to the Creative Commons license, and indicate if changes were made. The Creative Commons Public Domain Dedication waiver (http://creativecommons.org/publicdomain/zero/1.0/) applies to the data made available in this article, unless otherwise stated. 


\section{Introduction}

Lung cancer remains the leading cause of cancer-associated deaths globally. Non-small cell lung cancer (NSCLC) accounts for approximately 85\% of all lung cancer cases [1] and about 30\% of NSCLC patients have locally advanced diseases [2]. For unresectable, locally advanced NSCLC patients with good performance status, concurrent chemoradiotherapy (CCRT) remains a standard of care. A meta-analysis of individual patient data from nine randomised trials [3] has shown that platin-based CCRT improved survival compared to RT alone. Currently, etoposide-cisplatin (EP) and paclitaxel-cisplatin/carboplatin (PC) are two most common concurrent chemotherapy regimens [4-7]. However, the outcomes have remained unsatisfactory. Recently, a number of new generation chemotherapy agents such as vinorelbine [8-11], docetaxel [12-14], gemcitabine [12], irinotecan [15], pemetrexed [9, 16, 17], and S-1 $[10,14]$ are increasingly being used concurrently with thoracic radiation for locally advanced NSCLC patients, and have shown good efficacy in clinical trials. However, direct comparison trials between these new options and conventional regimens like EP are still lacking, and therefore, there are still unresolved questions around which is the optimal chemotherapy regimen used concurrently with thoracic radiation.

Network meta-analysis is the best way to solve aforementioned questions, which enable indirect comparisons to account for missing head-to-head data and multiple regimen comparisons. The study aimed to perform a network meta-analysis to estimate the relative efficacy and tolerability of different agents based concurrent chemotherapy regimens, attempting to identify the most preferable regimen used concurrently with thoracic radiation for locally advanced NSCLC.

\section{Materials and methods}

\section{Literature search strategy}

This meta-analysis was conducted in accordance with the Preferred Reporting Items for Systematic Reviews and Meta-analysis (PRISMA) criteria [18]. PubMed, Embase, Cochrane Library, Web of Science, and major international scientific meetings were searched for the available studies published before October 31, 2018, using the strategy as shown in Additional file 1: Table $\mathrm{S} 1$. The reference lists of retrieved studies were manually scanned for relevant additional studies missed by the electronic search.

\section{Inclusion and exclusion criteria}

Studies were included if they met the following criteria: (1) types of studies: randomized controlled trials (RCTs); (2) types of participants: participants with a histopathological diagnosis of locally advanced NSCLC; (3) types of interventions: one or more regimens for experimental arm, and the presence of a control for comparison; and (4) outcome: reported overall survival (OS) and/or progression-free survival (PFS) data. Studies were excluded if any of the following criteria were applied: (1) letters, editorials, case reports, and reviews; and (2) survival data could not be extracted from the literature.

\section{Data extraction}

The data were extracted by two investigators independently. The following data were extracted from each study: first author, years of publication, duration of the study, country of origin, treatments, numbers of patients (experimental arm/control arm), data of OS, PFS, objective response rate (ORR), and serious adverse events (SAEs).

\section{Quality assessment}

The methodological quality of RCTs was assessed by Cochrane risk of bias tool [19], which consists of the following five domains: sequence generation, allocation concealment, blinding, incomplete data, and selective reporting. A RCT was finally rated as "low risk of bias" (all key domains indicated as low risk), "high risk of bias" (one or more key domains indicated as high risk), and "unclear risk of bias".

\section{Statistical analysis}

The primary outcome was OS, and the secondary outcomes were PFS, ORR, and SAEs. Hazard ratios (HRs) or odds ratios (ORs) and their 95\% confidence intervals (CIs) were used as summary statistics. For direct comparisons, standard pairwise meta-analysis (PWMA) was performed. A statistical test for heterogeneity was performed using the chi-square $\left(\chi^{2}\right)$ and $I$-square $\left(I^{2}\right)$ tests with the significance set at $I^{2}>50 \%$ or $P<0.10$.

The Bayesian network-meta analysis (NMA) was performed in a random-effect model using Markov chain Monte Carlo methods [20, 21] in JAGS and the GeMTC package in $\mathrm{R}$ (https://rugis.org/software/r-packages/ gemtc). For each outcome measure, four independent Markov chains were simultaneously run for 20,000 burn-ins and 100,000 inference iterations per chain to obtain the posterior distribution. The traces plot and Brooks-Gelman-Rubin method were used to assess the convergence of model [22]. Treatment effects were estimated by HR/OR and corresponding 95\% CI.

Network consistency was assessed with node-split models by statistically testing between direct and indirect estimates within treatment loop [23]. To rank probabilities of all available treatments, the surfaces under the cumulative ranking curve (SUCRAs) were calculated [24]. SUCRA equals one if the treatment is certain to be the best and zero if it's certain to be the worst [24]. To 
jointly compare the efficacy and tolerability of each treatment and to assess their benefit-risk ratios, we ranked them based simultaneously on the SUCRA value of OS and tolerability (1-SUCRA $\mathrm{SAEs}_{\mathrm{SAs}}$ ) in the ranking plot. Lastly, comparison-adjusted funnel plot was used to detect the presence of small-study effects or publication bias [25].

\section{Results}

\section{Literature search results and characteristics of included studies}

The literature search results and study selection process are shown in Fig. 1. The initial search retrieved 5966 studies. After removing the duplicates, 1953 citations were identified, and 1889 of them were excluded through an abstract review. The remaining 64 studies were screened through a full-text review for further eligibility. Finally, 14 RCTs with 2975 patients randomized to receive the twelve categories of treatments were included in the meta-analysis. The twelve treatments were
EP, PC, pemetrexed-cisplatin/carboplatin (PP), S-1-cisplatin (SP), uracil/tegafur (UFT)-cisplatin (UP), vinorelbine-cisplatin (NP), gemcitabine-cisplatin (GP), docetaxel-cisplatin (DP), irinotecan-carboplatin (IC), mitomycin-vindesine-cisplatin (MVP), $\mathrm{PC}+$ cetuximab (PC-Cet), and PP + cetuximab (PP-Cet), respectively. One trial comparing cisplatin-pemetrexed vs. carboplatin-pemetrexed [26] was excluded because the two regimens were regarded as the same category of treatment (PP) in this meta-analysis. Two trials comparing SP vs. cisplatin alone were excluded due to that cisplatin alone is not commonly used clinically. Of the 14 included trials, twelve were two-arm studies, and the rest two was three-arm studies $[12,15]$. The study characteristics are shown in Table 1.

\section{Assessment of included trial}

The risk of bias in included RCTs was summarized in Additional file 2: Figure S1. Seven trials [5, 6, 8, 12, 14, $15,17]$ were judged to be unclear risk of bias, as they

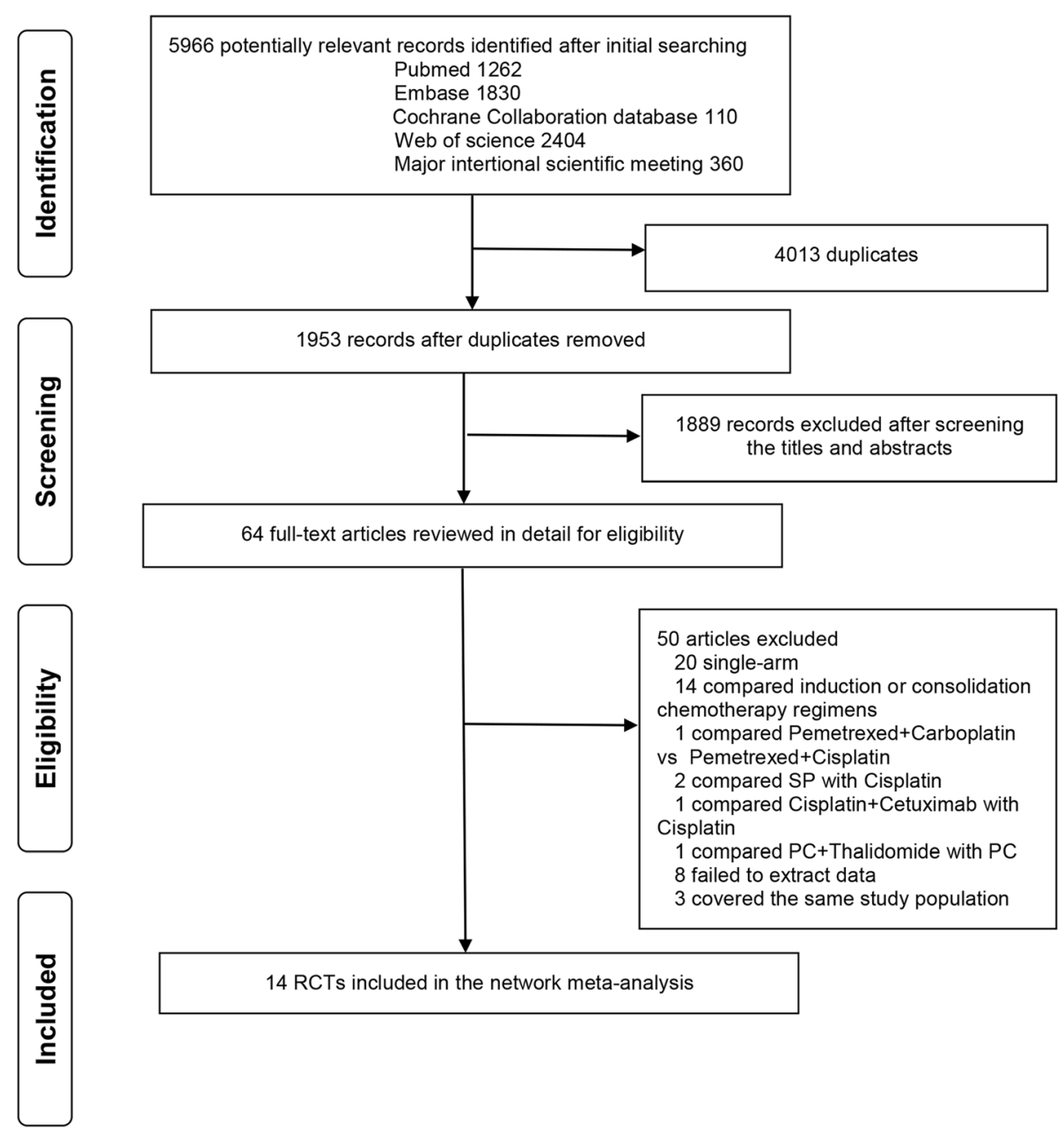

Fig. 1 Literature search and selection. SP, S-1-cisplatin; PC, paclitaxel-cisplatin/carboplatin 


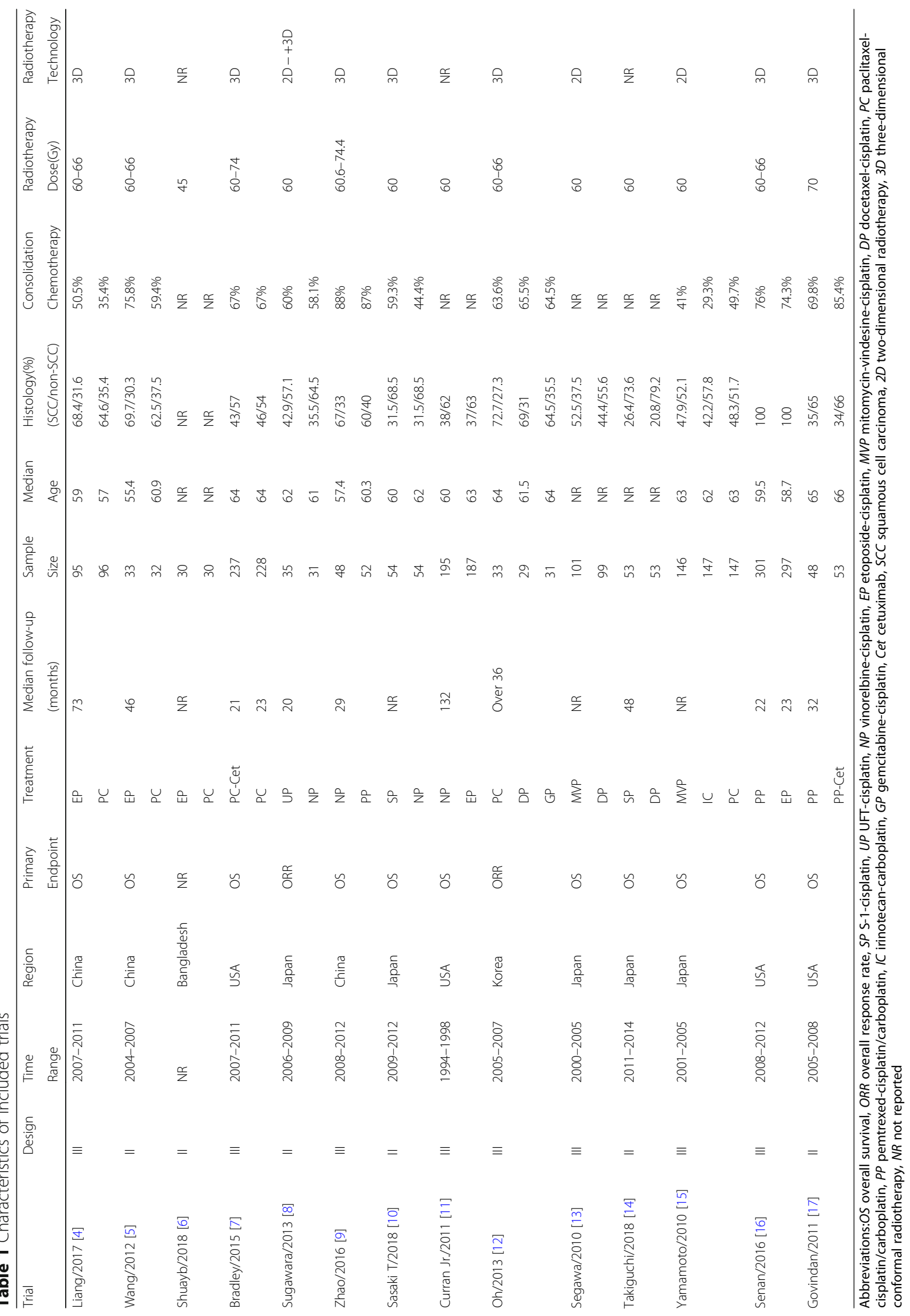


had more than three domains indicating as unclear risk. The remaining trials were rated with a low risk of bias. No trial was judged to be high risk of bias. Funnel plot analysis in term of OS did not indicate any evident risk of publication bias (Additional file 3: Figure S2).

\section{Conventional pairwise meta-analysis}

Results of single trial and direct comparison meta-analysis are shown in Table 2. Direct comparison meta-analysis was feasible only for EP vs. PC. EP was more effective than PC in terms of OS ( $\mathrm{HR}=0.85,95 \%$ CI: 0.77-0.94) and PFS (HR $=0.66,95 \% \mathrm{CI}: 0.47-0.95)$. No significant differences were observed in ORR and overall SAEs between the two arms. PC had a trend higher risk of causing grade $\geqq 3$ radiation pneumonitis (RP) than EP (OR $=0.48,95 \%$ CI: $0.21-1.1 ; P=0.08)$.

\section{Network meta-analysis}

The network plot established for NMA is shown in Fig. 2. Results of the NMA were presented in Additional file 4: Table S2. In term of OS, EP showed a trend significant advantage over $\mathrm{PC}(\mathrm{HR}=0.83$, 95\% CI: $0.65-1.0 ; P=$ $0.05)$. Other regimen comparisons did not produce statistically significant differences. With regard to PFS and ORR, no significant differences were observed for all regimen comparisons. As for overall SAEs and RP, MVP showed significantly higher risk of SAEs in comparison to each regimen except GP and PC-Cet. DP was more likely to cause SAEs than SP (OR $=0.51,95 \%$ CI: 0.30 $0.86)$ and UP (OR $=0.38,95 \% \mathrm{CI}: 0.15-0.96)$. NP resulted in a higher and a trend higher risk of SAEs than $\mathrm{UP}(\mathrm{OR}=0.47,95 \% \mathrm{CI}: 0.24-0.94)$ and $\mathrm{SP}(\mathrm{OR}=0.63$, 95\% CI: $0.38-1.0 ; P=0.05)$, respectively. $P C$ had a trend higher risk of grade $\geqq 3 \mathrm{RP}$ than $\mathrm{PP}(\mathrm{OR}=0.053,95 \% \mathrm{CI}$ : $0.00064-1.0 ; P=0.05)$ and $\mathrm{EP}(\mathrm{OR}=0.19,95 \% \mathrm{CI}$ : $0.016-1.1 ; P=0.06)$.

\section{Inconsistency assessment and treatment ranking}

There were three independent closed loops in the network for OS, ORR, overall SAEs: EP-NP-PP, PC-DP-MVP, and PC-DP-SP-NP-EP; two independent closed loops for PFS: PC-DP-MVP; and PC-DP-SP-NP-EP. Analysis of inconsistency showed that the NMA results were similar to the PWMA results for the four outcomes, which suggested the consistency between the direct and indirect evidence (Additional file 5: Figure S3).

The treatment rankings based on SUCRA are shown in Table 3. In terms of OS, SP was the most effective treatment $(0.80)$, followed by PP (0.70), PP-Cet (0.68), UP (0.68), NP (0.65), EP (0.60), and DP (0.60). With regard to PFS, PP was the most effective treatment (0.89), followed by PP-Cet (0.86), EP (0.75), and UP (0.74). As for overall SAEs, UP was ranked as the least toxic regimen (0.03), followed by SP (0.11) and PP (0.33); MVP (1.0) was ranked as the highest toxic regimen. In term of RP, PC-Cet (0.83), PC (0.69) were ranked the highest and second-highest risk of causing grade $\geqq 3 \mathrm{RP}$ respectively.

To further assess the benefit-risk ratios of the twelve treatments simultaneously, we ranked them based on the SUCRA values of OS and tolerability (1-SUCRA ${ }_{\text {SAE }}$ ) in the ranking plot (Fig. 3). SP was likely to be the optimal because it had the most efficacy with low risk of causing SAEs. UP, PP, and PP-Cet were ranked the second, third, and fourth respectively. EP and NP also had good efficacy with moderate risk of causing SAEs, and were ranked the fifth and sixth respectively. DP had similar efficacy to EP but with higher risk of causing SAEs. GP and PC-Cet appeared to be the worst and second worst for their poor efficacy and poor tolerability.

\section{Discussion}

To our knowledge, this is the first network meta-analysis assessing the comparative efficacy and tolerability of all major chemotherapy regimens used concurrently with thoracic radiation for patients with locally advanced NSCLC. It showed that SP was likely to be the most preferable regimen based on the benefit-risk ratio. S-1 is a new oral fluoropyrimidine formulation that comprises tegafur, 5-chloro-2, 4-dihydroxypyridine, and potassium oxonate. Data from several single-arm phase II trials [27-29] have consistently shown that SP with concurrent thoracic radiation is a promising treatment for patients with locally advanced NSCLC, with OS rate of $51-70 \%$ at 2 years and $43-52.9 \%$ at 5 years, which appear to be superior to other concurrent chemotherapy regimens employed in other clinical trials. In a more recent randomized phase II trial, SP provided higher 2 years OS rate $(75.6 \%$ vs. $68.5 \%)$ and longer PFS (14.8 months vs. 12.3 months) than NP [10]. Although there was no statistically significant difference, the PFS curve showed more favourable results for the SP arm over the long term, and tolerability was better. Similarly, SP resulted in a superior 2, 5-years OS with less toxicity compared with DP in another recent randomized phase II trial of SP or DP with concurrent thoracic radiotherapy for inoperable stage III NSCLC [14]. However, to date SP has not been compared directly with other established regimens. In our NMA, SP had the highest probability $(80 \%)$ of being the most effective treatment in improving OS and with a low risk of causing SAEs, suggesting it to be a promising candidate as a standard regimen for locally advanced NSCLC. Besides, UFT (another oral fluoropyrimidine formulation) plus cisplatin (UP) also showed a good efficacy with better tolerability in the present NMA. Nevertheless, S-1 is not approved by the FDA. While data from 2 randomized phase II trials 
Table 2 Results of single trial and direct comparison meta-analysis

\begin{tabular}{|c|c|c|c|c|c|c|c|c|c|c|c|}
\hline \multirow[t]{2}{*}{ Treatment } & \multirow[t]{2}{*}{ Study } & \multirow{2}{*}{$\begin{array}{l}\text { OS } \\
\mathrm{HR}(95 \% \mathrm{Cl})\end{array}$} & \multirow{2}{*}{$\begin{array}{l}\text { PFS } \\
\mathrm{HR}(95 \% \mathrm{Cl})\end{array}$} & \multirow{2}{*}{$\begin{array}{l}\text { ORR } \\
\text { OR(95\% Cl) }\end{array}$} & \multirow{2}{*}{$\begin{array}{l}\text { Overall SAEs } \\
\text { OR(95\% Cl) }\end{array}$} & \multirow{2}{*}{$\begin{array}{l}\text { RP } \\
\text { OR(95\% Cl) }\end{array}$} & \multicolumn{5}{|c|}{ Heterogeneity $I^{2}(\%)$} \\
\hline & & & & & & & OS & PFS & ORR & SAE & $\mathrm{RP}$ \\
\hline EP vs PC & {$[4-6]$} & $0.85(0.77-0.94)$ & $0.66(0.47-0.95)$ & $1.0(0.86-1.2)$ & $1.2(0.81-1.4)$ & $0.48(0.21-1.1)$ & 0 & 0 & 59 & 0 & 27 \\
\hline PC-Cet vs PC & [7] & $1.1(0.84-1.4)$ & $0.99(0.8-1.2)$ & $N R$ & $1.2(1.1-1.4)$ & $0.56(0.27-1.2)$ & & & & & \\
\hline UP vs NP & [8] & $0.86(0.35-2.1)$ & $0.68(0.35-1.3)$ & $1.6(0.53-5.1)$ & $0.47(0.29-0.76)$ & $0.88(0.12-6.6)$ & & & & & \\
\hline NP vs PP & [9] & $1.7(0.81-3.4)$ & $1.6(0.91-2.6)$ & $1.4(0.38-5.4)$ & $1.9(1.1-3.3)$ & $5.7(0.26-120.6)$ & & & & & \\
\hline SP vs NP & [10] & $0.85(0.49-1.5)$ & $0.37(0.15-0.94)$ & $0.79(0.31-2.0)$ & $0.67(0.50-0.88)$ & $1.3(0.32-5.0)$ & & & & & \\
\hline NP vs EP & [11] & $0.93(0.75-1.1)$ & NR & $1.3(0.82-1.9)$ & $0.91(0.81-1.0)$ & $0.86(0.50-1.5)$ & & & & & \\
\hline PC vs DP & [12] & $1.0(0.33-3.3)$ & $1.1(0.48-2.3)$ & $0.67(0.23-2.0)$ & $0.67(0.39-1.2)$ & $4.7(0.22-101.6)$ & & & & & \\
\hline PC vs GP & [12] & $0.77(0.34-1.8)$ & $0.67(0.3-1.5)$ & $1.1(0.40-3.0)$ & $0.80(0.45-1.4)$ & $1.9(0.17-22.5)$ & & & & & \\
\hline DP vs GP & [12] & $0.65(0.28-1.5)$ & $0.72(0.36-1.5)$ & $1.7(0.56-4.9)$ & $1.2(0.70-2.0)$ & $0.34(0.01-8.8)$ & & & & & \\
\hline MVP vs DP & [13] & $1.2(0.8-1.7)$ & $1.2(0.89-1.7)$ & $0.64(0.33-1.2)$ & $1.8(1.5-2.0)$ & $0.67(0.24-1.8)$ & & & & & \\
\hline SP vs DP & [14] & $0.81(0.39-1.7)$ & $1.1(0.63-1.9)$ & $1.2(0.52-2.8)$ & $0.49(0.35-0.68)$ & $0.10(0.31-3.2)$ & & & & & \\
\hline MVP vs IC & [15] & $0.98(0.74-1.3)$ & $0.89(0.69-1.1)$ & $1.5(0.95-2.5)$ & $1.8(1.5-2.0)$ & $0.33(0.06-1.6)$ & & & & & \\
\hline MVP vs PC & {$[15]$} & $0.95(0.72-1.3)$ & $1.1(0.82-1.4)$ & $1.2(0.71-1.9)$ & $2.1(1.8-2.4)$ & $0.33(0.06-1.6)$ & & & & & \\
\hline IC vs PC & [15] & $1.1(0.79-1.4)$ & $1.1(0.86-1.5)$ & $0.75(0.47-1.2)$ & $1.2(1.0-1.4)$ & $1.0(0.31-3.2)$ & & & & & \\
\hline PP vs EP & [16] & $0.98(0.79-1.2)$ & $0.86(0.71-1.0)$ & $1.1(0.81-1.6)$ & $0.96(0.85,1.1)$ & $0.68(0.21-2.2)$ & & & & & \\
\hline PP vs PP-Cet & {$[17]$} & $1.1(0.58-2.0)$ & $1.1(0.62-1.8)$ & $1.3(0.54-3.3)$ & $0.99(0.74-1.3)$ & $1.1(0.32-3.6)$ & & & & & \\
\hline
\end{tabular}

Abbreviations: OS overall survival, $P F S$ progression-free survival, ORR objective response rate, SAEs serious adverse events, $R P$ radiation pneumonitis, $H R$ hazard ratio, $\mathrm{Cl}$ confidence interval, $O R$ odds ratio, $E P$ etoposide-cisplatin, $P C$ paclitaxel-cisplatin/carboplatin, UP uracil/tegafur(UFT)-cisplatin, NP vinorelbine-cisplatin, $P P$ pemetrexed-cisplatin/carboplatin, SP S-1-cisplatin, DP docetaxel-cisplatin, GP gemcitabine-cisplatin, MVP mitomycin-vindesine-cisplatin, IC irinotecan-carboplatin, Cet cetuximab, NR not reported

are promising regarding the use or S-1-cisplatin for CCRT in locally advanced NSCLC, a phase III trial is warranted before any recommendations regarding its use in this setting can be made.

PP has been the standard first-line treatment option in patients with metastatic non-squamous NSCLC. However, no clear survival advantages are reported for locally advanced case. A phase III trial (PROCLAIM) compared concurrent chemoradiation using PP vs. EP in stage III non-squamous NSCLC [16]. No statistically significant difference in OS was observed. Median PFS were 11.4 and 9.8 respectively, trended in favor of PP. However, PP had significantly lower Grade 3 or higher SAEs compared to EP $(P=0.01)$. In our NMA, PP was ranked third-best regimen in term of the benefit-risk ratio. The addition of Cetuximab did not demonstrate a survival advantage compared with PP alone. It should be noted that all included patients (except PROCLAIM trial) were with mixed histological types. In a phase III trial comparing PP and GP in patients with advanced NSCLC, PP resulted in a superior OS in non-squamous patients but did a worse survival for patients with squamous histology tumors compared with GP [30]. Further head-to-head comparisons, for locally advanced disease, with other concurrent regimens according to histological type are also needed.
EP and PC, two most common regimens administered currently with radiation to date, have recently been compared directly in several clinic trials. In a most recent multicenter randomized phase III trial [4] comparing $\mathrm{EP}$ and $\mathrm{PC}$ with concurrent thoracic radiotherapy in unresectable stage III NSCLC, the 3-year OS was significantly higher in the EP arm than in the PC arm. Similar survival advantage in EP arm was also found in two phase II trials $[5,6]$. In our NMA, EP failed to show survival advantage compared with any other regimens though with $\mathrm{PC}$. Based on the benefit-risk ratio, EP showed better efficacy but with moderate risk of causing SAEs, and were ranked sixth-best after SP, UP, PP, and NP.

$\mathrm{RP}$ remains one of the most common side effects for patients treated with chemotherapy concurrently with thoracic radiation, and different chemotherapy regimens have been reported to be related to the differences in RP risk $[5,31]$. In a phase II trial of concurrent EP or PC and thoracic radiotherapy for stage III NSCLC [5], the rate of grade $\geq 2 \mathrm{RP}$ was $25 \%$ in the $\mathrm{PE}$ arm and $48.5 \%$ in the $\mathrm{PC}$ arm $(P=0.09)$. Data from a large meta-analysis of predictors of RP showed that concurrent $\mathrm{PC}$ resulted in five times the risk of grade $\geq 2 \mathrm{RP}$ compared with EP [31]. In our NMA, $\mathrm{PP}$ and $\mathrm{PC}$ were ranked the lowest and highest risk of causing grade $\geq 3 \mathrm{RP}$, respectively. Other regimens 


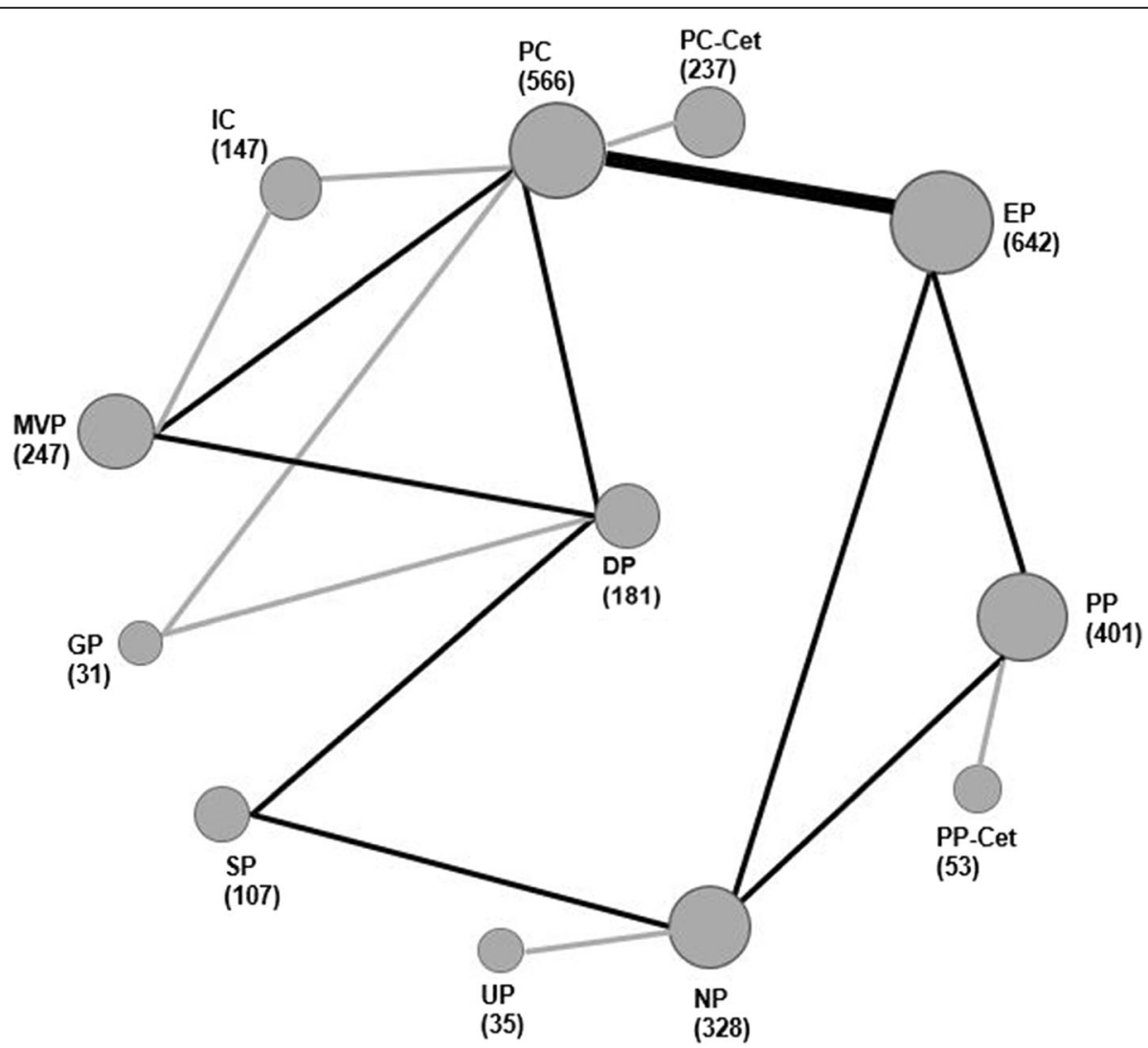

Fig. 2 Network of eligible comparisons for the Bayesian network meta-analysis. The size of the nodes is proportional to the number of patients (in parentheses) randomized to receive the treatment. The width of the lines is proportional to the number of trials (beside the line) comparing the connected treatments. EP, etoposide-cisplatin; PC, paclitaxel-cisplatin/carboplatin; SP, S-1-cisplatin; UP, uracil/tegafur (UFT)-cisplatin; PP, pemtrexed-cisplatin/carboplatin; NP, vinorelbine-cisplatin; DP, docetaxel-cisplatin; IC, irinotecan-carboplatin; GP, gemcitabine-cisplatin; MVP, mitomycin-vindesine-cisplatin; Cet, cetuximab

Table 3 SUCRA values for four outcomes

\begin{tabular}{|c|c|c|c|c|c|c|c|}
\hline \multicolumn{2}{|l|}{ OS } & \multicolumn{2}{|l|}{ PFS } & \multicolumn{2}{|l|}{ SAES } & \multicolumn{2}{|l|}{$\mathrm{RP}$} \\
\hline Treatment & SUCRA & Treatment & SUCRA & Treatment & SUCRA & Treatment & SUCRA \\
\hline $\mathrm{SP}$ & 0.80 & $\mathrm{PP}$ & 0.89 & UP & 0.03 & MVP & 0.32 \\
\hline PP & 0.70 & PP-Cet & 0.86 & $\mathrm{SP}$ & 0.11 & PP & 0.36 \\
\hline PP-Cet & 0.68 & EP & 0.75 & PP & 0.33 & PP-Cet & 0.37 \\
\hline UP & 0.68 & UP & 0.74 & PP-Cet & 0.38 & $\mathrm{NP}$ & 0.41 \\
\hline NP & 0.65 & $\mathrm{DP}$ & 0.55 & $P C$ & 0.41 & UP & 0.42 \\
\hline EP & 0.60 & SP & 0.48 & $\mathrm{NP}$ & 0.50 & $\mathrm{DP}$ & 0.46 \\
\hline DP & 0.60 & $\mathrm{NP}$ & 0.41 & EP & 0.50 & EP & 0.47 \\
\hline MVP & 0.35 & PC-Cet & 0.36 & GP & 0.60 & GP & 0.47 \\
\hline PC & 0.29 & $P C$ & 0.35 & PC-Cet & 0.69 & SP & 0.50 \\
\hline IC & 0.28 & MVP & 0.28 & IC & 0.7 & IC & 0.69 \\
\hline PC-Cet & 0.22 & IC & 0.17 & $\mathrm{DP}$ & 0.75 & $P C$ & 0.69 \\
\hline GP & 0.15 & GP & 0.15 & MVP & 1.0 & PC-Cet & 0.83 \\
\hline
\end{tabular}

Abbreviations: OS overall survival, $P F S$ progression-free survival, $S A E$ serious adverse events, $R P$ radiation pneumonitis, SUCRA surface under the cumulative ranking curve, EP etoposide-cisplatin, $P C$ paclitaxel-cisplatin/carboplatin, UP uracil/tegafur(UFT)-cisplatin, NP vinorelbine-cisplatin, $P P$ pemetrexed-cisplatin/carboplatin, SP S1-cisplatin, DP docetaxel-cisplatin, GP gemcitabine-cisplatin, MVP mitomycin-vindesine-cisplatin, IC irinotecan-carboplatin, Cet cetuximab 


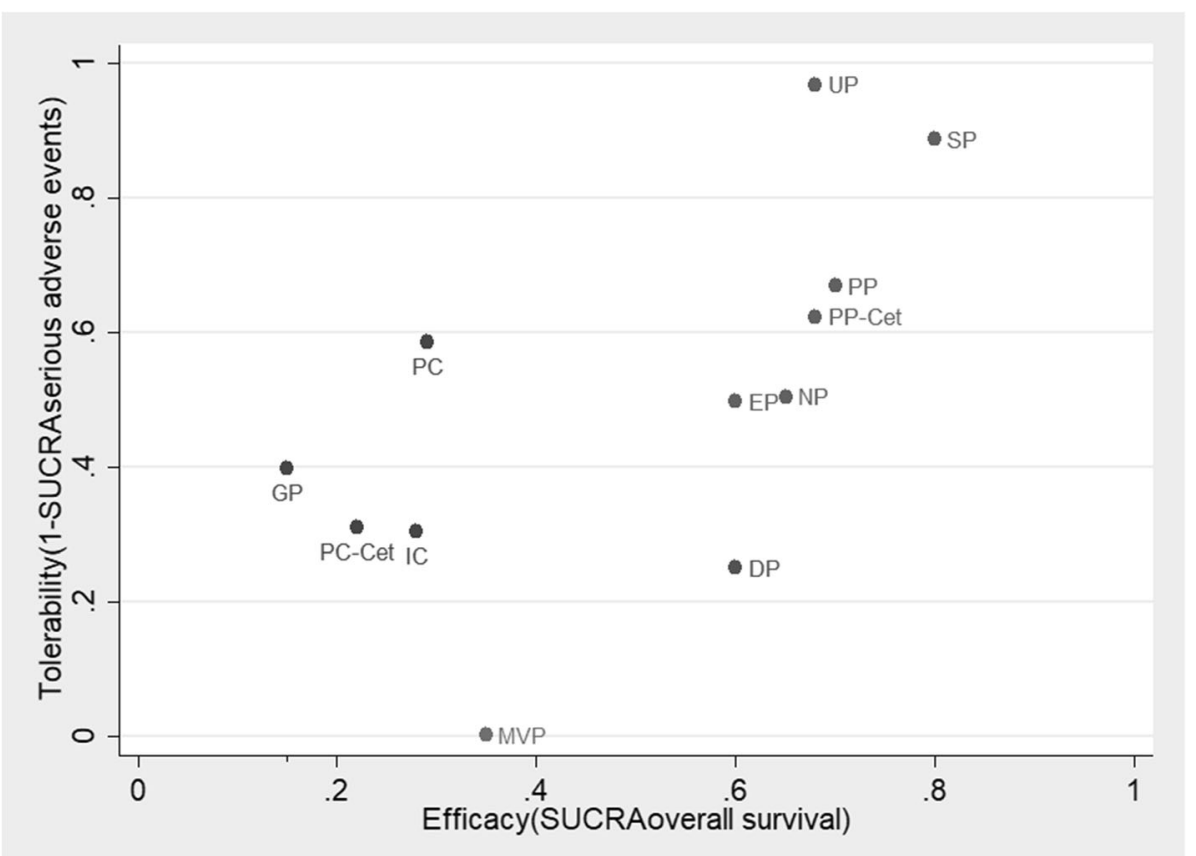

Fig. 3 Ranking plot based simultaneously on efficacy ( $x$-axis: SUCRA value of overall survival) and tolerability (y-axis: 1-SUCRA value of serious adverse events). SUCRA, surface under the cumulative ranking curves; SP, S-1-cisplatin; UP, uracil/tegafur (UFT)-cisplatin; PP, pemtrexed-cisplatin/ carboplatin; EP, etoposide-cisplatin; PC, paclitaxel-cisplatin/carboplatin; NP, vinorelbine-cisplatin; DP, docetaxel-cisplatin; IC, irinotecan-carboplatin; GP, gemcitabine-cisplatin; MVP, mitomycin-vindesine-cisplatin; Cet, cetuximab

such as NP, UP, DP, EP, GP, and SP, were with similarly moderate risk of causing grade $\geq 3 \mathrm{RP}$.

Molecularly targeted agents and immunotherapy have a defined role for metastatic NSCLC. The efficacy of these new classes of agents for locally advanced disease is undergoing investigation. In the 2017 Annual Meeting of the American Society of Clinical Oncology, Yu et al. reported a multicenter phase II trial of erlotinib vs. EP with concurrent thoracic radiotherapy for stage III NSCLC with epidermal growth factor receptor activating mutation [32]. Comparing with EP, median PFS of erlotinib arm was significantly improved (27.86 vs. 6.41 months, $P<0.001)$. However, the sample size is relatively small (21 in EP arm and 20 in erlotinib arm). These findings need a large sample size of phase III study to confirm. Morever, a recent phase III trial (PACIFIC) [33] compared PD-L1 inhibitor durvalumab with placebo in patients with stage III unresectable NSCLC, not progressing after chemoradiotherapy. The main chemotherapy regimens used for CCRT were cisplatin or carboplatin based regimens; in addition, $25.8 \%$ of the patients in the durvalumab group and $28.7 \%$ of those in the placebo group had received induction chemotherapy before definitive chemoradiotherapy. Patients receiving durvalumab had a three-fold increase in median PFS, and with a reduction in the risk of progression of $48 \%$. Although the PACIFIC trial has demonstrated the obvious advantage of consolidation durvalumab after chemoradiation, identification of patients who really benefit from the addition of durvalumab and the optimal concurrent chemotherapy regimen in combine with consolidation immune checkpoint inhibitor are warranted.

This network meta-analysis has a number of limitations. Firstly, in common with other meta-analyses, data were collected and analyzed basis of results reported from trials, instead of individual patient data. Secondly, all studies except PROCLAIM trial included patients with mixed histological types. Thus, we could not assess survival differences between regimens according to histological types. Thirdly, different toxicity criteria were used, and radiotherapy features (such as counturing, planning and delivery) were inconsistent in individual RCTs. Moreover, chemotherapy regimens used in different countries were studied togheter in the meta-analysis. These might lead to heterogeneity and inconsistency. Finally, some HRs of OS or PFS were calculated from the Kaplan-Meier curve due to that they were not directly reported in the articles. This may result in bias.

\section{Conclusions}

Based on efficacy and tolerability, SP is likely to be the most preferable regimen used concurrently with thoracic radiation for locally advanced NSCLC, followed by UP and PP. GP and PC-Cet appeared to be the worst and 
second-worst regimens for this population. Further direct head-to-head, well-designed, prospective studies are needed to confirm these findings.

\section{Additional files}

Additional file 1: Table S1. Search strategy (DOC $62 \mathrm{~kb}$ )

Additional file 2: Figure S1. Assessment of risk of bias. A: Methodological quality graph: authors' judgment about each methodological quality item presented as percentages across all included studies; B: Methodological quality summary: authors' judgment about each methodological quality item for each included study, "+" low risk of bias; "?" unclear risk of bias; "-" high risk of bias. (JPEG 17793 kb)

Additional file 3: Figure S2. Comparison-adjusted funnel plots of publication bias test for overall survival. SP, S-1-cisplatin; UP, uracil/tegafur (UFT)-cisplatin; PP, pemtrexed-cisplatin/carboplatin; EP, etoposidecisplatin; PC, paclitaxel-cisplatin/carboplatin; NP, vinorelbine-cisplatin; DP, docetaxel-cisplatin; IC, irinotecan-carboplatin; MVP, mitomycin-vindesinecisplatin; GP, gemcitabine-cisplatin; Cet, cetuximab. (JPG 6940 kb)

Additional file 4: Table S2. Results of network meta-analysis (DOC $117 \mathrm{~kb}$ )

Additional file 5: Figure S3. Inconsistency evaluation by node-splitting analyses. (a) overall survival; (b) progression-free survival; (c) objective response rate; (d) serious adverse events. $E P$, etoposide-cisplatin; $P C$, paclitaxel-cisplatin/carboplatin; PP, pemtrexed-cisplatin/carboplatin; MVP, mitomycin-vindesine-cisplatin; SP, S-1-cisplatin; NP, vinorelbine-cisplatin; DP, docetaxel-cisplatin. (JPG $9280 \mathrm{~kb}$ )

\section{Abbreviations}

CCRT: Concurrent chemoradiotherapy; Cl: Confidence interval; DP: Docetaxelcisplatin; EP: Etoposide-cisplatin; GP: Gemcitabine-cisplatin; HR: Hazard ratio; IC: Irinotecan-carboplatin; MVP: Mitomycin-vindesine-cisplatin; NMA: Networkmeta analysis; NP: Vinorelbine-cisplatin; NSCLC: Non-small cell lung cancer; OR: Odds ratio; ORR: Objective response rate; OS: Overall survival; PC: Paclitaxel-cisplatin/carboplatin; PC-Cet: PC + cetuximab; PFS: Progressionfree survival; PP: Pemetrexed-cisplatin/carboplatin; PP-Cet: PP + cetuximab; PWMA: Pairwise meta-analysis; RCT: Randomized controlled trial; RP: Radiation pneumonitis; SAE: Serious adverse event; SP: S-1-cisplatin; SUCRA: Surfaces under the cumulative ranking curve; UFT: Uracil/tegafur; UP: Uracil/tegafur-cisplatin

\section{Acknowledgements}

None.

\section{Funding}

Present study did not receive any funding.

\section{Availability of data and materials}

Not applicable.

\section{Authors' contributions}

JD had full access to all the data and takes responsibility for the integrity of the data and the accuracy of the data analysis. Study concept and design: $J \mathrm{D}, \mathrm{TL}, \mathrm{ZH}$. Acquisition of data: $\mathrm{TL}, \mathrm{ZH}$. Analysis and interpretation of data: $\mathrm{TL}$, $\mathrm{ZH}$. Drafting of the manuscript: TL, ZH, GL. Critical revision of the manuscript for important intellectual content: JD. Statistical analysis: TL, ZH. All authors read and approved the final manuscript.

\section{Ethics approval and consent to participate}

There was no ethics approval necessary because in this meta-analysis we were pulling numbers from the published manuscripts and pooling results.

\section{Consent for publication}

Not applicable.

\section{Competing interests}

The authors declare that they have no competing interests.

\section{Publisher's Note}

Springer Nature remains neutral with regard to jurisdictional claims in published maps and institutional affiliations.

Received: 8 December 2018 Accepted: 14 February 2019

Published online: 29 March 2019

\section{References}

1. Siegel RL, Miller KD, Jemal A. Cancer statistics, 2017. CA Cancer J Clin. 2017; 67:7-30.

2. Yang P, Allen MS, Aubry MC, et al. Clinical features of 5,628 primary lung cancer patients: experience at Mayo Clinic from 1997 to 2003. Chest. 2005; 128:452-62.

3. Aupérin A, Le Péchoux C, Pignon JP, et al. Concomitant radiochemotherapy based on platin compounds in patients with locally advanced non-small cell lung cancer (NSCLC): a meta-analysis of individual data from 1764 patients. Ann Oncol. 2006;17:473-83.

4. Liang J, Bi N, Wu S, et al. Etoposide and cisplatin versus paclitaxel and carboplatin with concurrent thoracic radiotherapy in unresectable stage III non-small cell lung cancer: a multicenter randomized phase III trial. Ann Oncol. 2017;28:777-83

5. Wang L, Wu S, Ou G, et al. Randomized phase II study of concurrent cisplatin/ etoposide or paclitaxel/carboplatin and thoracic radiotherapy in patients with stage III non-small cell lung cancer. Lung Cancer. 2012;77:89-96.

6. Shuayb M, Shahi MSJR, Hossen MM. Cisplatin/etoposide or paclitaxel/ carboplatin with concurrent radiation therapy in stage IIIB non-small cell lung cancer: A one-year phase II trial at a low resource setting. J Thorac Oncol. 2018;13:4 Supplement 1 (S66).

7. Bradley JD, Paulus R, Komaki R, et al. Standard-dose versus high-dose conformal radiotherapy with concurrent and consolidation carboplatin plus paclitaxel with or without cetuximab for patients with stage IIIA or IIIB nonsmall-cell lung cancer (RTOG 0617): a randomised, two-by-two factorial phase 3 study. Lancet Oncol. 2015;16:187-99.

8. Sugawara S, Maemondo M, Tachihara M, et al. Randomized phase II trial of uracil/tegafur and cisplatin versus vinorelbine and cisplatin with concurrent thoracic radiotherapy for locally advanced unresectable stage III non-smallcell lung cancer: NJLCG 0601. Lung Cancer. 2013;81:91-6.

9. Zhao Q, Wang Z, Huang W, et al. Phase III study of cisplatin with pemtrexed or vinorelbine plus concurrent late course accelerated hyperfractionated radiotherapy in patients with unresectable stage III non-small cell lung cancer. Oncotarget. 2016;7:8422-31.

10. Sasaki T, Seto T, Yamanaka T, et al. A randomised phase II trial of S-1 plus cisplatin versus vinorelbine plus cisplatin with concurrent thoracic radiotherapy for unresectable, locally advanced non-small cell lung cancer: WJOG5008L. Br J Cancer. 2018;119:675-82.

11. Curran WJ Jr, Paulus R, Langer CJ, et al. Sequential vs. concurrent chemoradiation for stage III non-small cell lung cancer: randomized phase III trial RTOG 9410. J Natl Cancer Inst. 2011;103:1452-60.

12. Oh IJ, Kim KS, Kim YC, et al. A phase III concurrent chemoradiotherapy trial with cisplatin and paclitaxel or docetaxel or gemcitabine in unresectable non-small cell lung cancer: KASLC 0401. Cancer Chemother Pharmacol. 2013;72:1247-54.

13. Segawa Y, Kiura K, Takigawa N, et al. Phase III trial comparing docetaxel and cisplatin combination chemotherapy with mitomycin, vindesine, and cisplatin combination chemotherapy with concurrent thoracic radiotherapy in locally advanced non-small-cell lung cancer: OLCSG 0007. J Clin Oncol. 2010;28:3299-306.

14. Takiguchi $Y$, Yamada $\mathrm{K}$, Tanaka $\mathrm{H}$, Kubota $\mathrm{K}$, et al. Survival update in randomized phase II trial of S-1/cisplatin (SP) or docetaxel/cisplatin (DP) with concurrent thoracic radiotherapy for inoperable stage III non-small cell lung cancer (NSCLC)-TORG1018. Ann Oncol. 2018;29:suppl_8 1 October 2018, mdy291.009.

15. Yamamoto N, Nakagawa K, Nishimura Y, et al. Phase III study comparing second- and third-generation regimens with concurrent thoracic radiotherapy in patients with unresectable stage III non-small-cell lung cancer: West Japan thoracic oncology group WJTOG0105. J Clin Oncol. 2010;28:3739-45.

16. Senan S, Brade A, Wang LH, et al. PROCLAIM: randomized phase III trial of Pemetrexed-cisplatin or etoposide-cisplatin plus thoracic radiation therapy followed by consolidation chemotherapy in locally advanced nonsquamous non-small-cell lung Cancer. J Clin Oncol. 2016;34:953-62. 
17. Govindan R, Bogart J, Stinchcombe T, et al. Randomized phase II study of pemetrexed, carboplatin, and thoracic radiation with or without cetuximab in patients with locally advanced unresectable non-small-cell lung cancer: Cancer and Leukemia Group B trial 30407. J Clin Oncol. 2011;29:3120-5.

18. Moher D, Liberati A, Tetzlaff J, et al. Preferred reporting items for systematic reviews and meta-analyses: the PRISMA statement. Int I Surg. 2010;8:336-41.

19. Higgins JP, Altman DG, Gøtzsche PC, et al. The Cochrane Collaboration's tool for assessing risk of bias in randomised trials. BMJ. 2011;343:d5928.

20. Gelman A, Rubin D. Inference from iterative simulation using multiple sequences. Stat Sci. 1992;7:457-511.

21. Neupane B, Richer D, Bonner AJ, et al. Network meta-analysis using R: a review of currently available automated packages. PLoS One. 2014;9:e115065

22. Brooks S, Gelman A. General methods for monitoring convergence of iterative simulations. J Comput Graph Stat. 1998;7:434-55.

23. van Valkenhoef $\mathrm{G}$, Dias $\mathrm{S}$, Ades $\mathrm{AE}$, et al. Automated generation of nodesplitting models for assessment of inconsistency in network meta-analysis. Res Synth Methods. 2016;7:80-93.

24. Chaimani A, Higgins JP, Mavridis D, et al. Graphical tools for network metaanalysis in STATA. PLoS One. 2013;8:e76654.

25. Egger M, Davey Smith G, Schneider M, et al. Bias in meta-analysis detected by a simple, graphical test. BMJ. 1997;315:629-34.

26. Choy H, Schwartzberg LS, Dakhil SR, et al. Phase 2 study of pemetrexed plus carboplatin, or pemetrexed plus cisplatin with concurrent radiation therapy followed by pemetrexed consolidation in patients with favorableprognosis inoperable stage IIIA/B non-small-cell lung cancer. J Thorac Oncol. 2013;8:1308-16.

27. Kaira K, Tomizawa Y, Yoshino R, et al. Phase II study of oral S-1 and cisplatin with concurrent radiotherapy for locally advanced non-small-cell lung cancer. Lung Cancer. 2013;82:449-54.

28. Taira T, Yoh K, Nagase S, et al. Long-term results of S-1 plus cisplatin with concurrent thoracic radiotherapy for locally advanced non-small-cell lung cancer. Cancer Chemother Pharmacol. 2018;81:565-72.

29. Ichinose Y, Seto T, Sasaki T, et al. S-1 plus cisplatin with concurrent radiotherapy for locally advanced non-small cell lung cancer: a multiinstitutional phase II trial (West Japan thoracic oncology group 3706). J Thorac Oncol. 2011;6:2069-75.

30. Scagliotti GV, Parikh P, von Pawel J, et al. Phase III study comparing cisplatin plus gemcitabine with cisplatin plus pemetrexed in chemotherapy-naive patients with advanced-stage non-small-cell lung cancer. J Clin Oncol. 2008; 26:3543-451.

31. Palma DA, Senan $S$, Tsujino $K$, et al. Predicting radiation pneumonitis after chemoradiation therapy for lung cancer: an international individual patient data meta-analysis. Int J Radiat Oncol Biol Phys. 2013;85:444-50.

32. Xing $L, W u G$, Wang $L$, et al. A multicenter, randomized, open-label, phase II trial of erlotinib versus etoposide plus cisplatin with concurrent radiotherapy in unresectable stage III non-small cell lung cancer (NSCLC) with epidermal growth factor receptor (EGFR) activating mutation. J Clin Oncol. 2017;35:15 Supplement 1.

33. Antonia SJ, Villegas A, Daniel D, et al. Durvalumab after chemoradiotherapy in stage III non-small-cell lung cancer. N Engl J Med. 2017;377:1919-29.

Ready to submit your research? Choose BMC and benefit from:

- fast, convenient online submission

- thorough peer review by experienced researchers in your field

- rapid publication on acceptance

- support for research data, including large and complex data types

- gold Open Access which fosters wider collaboration and increased citations

- maximum visibility for your research: over $100 \mathrm{M}$ website views per year

At BMC, research is always in progress.

Learn more biomedcentral.com/submissions 